\title{
„Praxen fit für den Gesundheitsmarkt machen"
}

\begin{abstract}
Dass der freiberufliche Zahnarzt immer auch ein guter Unternehmer sein muss, um seine Praxis (über)lebensfähig zu halten, hat der FVDZ schon früh verstanden. Mit dem Thema Praxisökonomie setzt sich Bundesvorstandsmitglied Dr. Wolfram Sadowski deshalb nicht erst seit zwei Jahren auseinander. Im Bundesvorstand laufen bei ihm die ökonomischen Fäden zusammen - als ausgewiesener Haushaltsexperte ist er der "Finanzminister" im Vorstandskollegium.
\end{abstract}

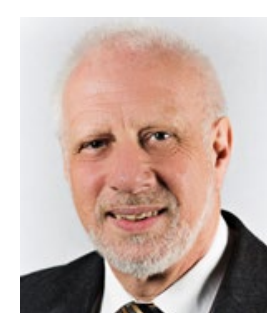

\section{Wo stand der Verband, als Sie den Bereich Ökonomie übernommen haben?}

Im Bereich Ökonomie hatte der Verband auch vor der Wahl 2013 einiges für seine Mitglieder zu bieten. Der FVDZ hat ja schon relativ früh erkannt, dass die betriebswirtschaftliche und Praxismanagement-Themen für den freiberuflichen Zahnarzt enorm wichtig sind. Wenn man als Zahnarzt vergangener Generation etwa nur wissen musste, wie viel Steuern man zu zahlen hat, so sind die Erfordernisse in heutiger Zeit wesentlich höher. Es gab also schon Angebote, die der Verband seinen Mitgliedern machte, aber die waren bei weitem nicht so strukturiert und aktuell, wie das heute der Fall ist. Zahnärzte müssen sich ja mit ganz neuen Fragen beschäftigen, wie neue Praxisstrukturen, weitere gesetzliche Pflichten, Gewinnermittlung und -verteilung in Berufsausübungsgemeinschaften und vieles mehr.

\section{Was haben Sie in den vergangenen zwei Jahren erreicht?}

Zunächst haben wir alle Angebote, die wir zur Praxisökonomie hatten und diejenigen, die neu dazu gekommen sind, unter der Dachmarke „FVDZ Ökonomie“ gebündelt, so dass jeder Interessierte sich gleich zurechtfindet. Aufgebaut haben wir jetzt eine ganze Kaskade von Möglichkeiten, sich betriebswirtschaftlich fit zu machen. Bei jedem der vier Kongresse des FVDZ werden Fortbildungsmöglichkeiten zum Praxismanagement angeboten nicht nur beim ausgewiesen Praxisökonomie-Kongress auf Sylt.

Außerdem wird das Beratungsangebot des FVDZ erweitert und ein Steuerberater-Netzwerk aufgebaut, für das ausschließlich auf die zahnärztlichen Probleme spezialisierte Steuerberater tätig sind. Drängende Probleme können direkt über eine Hotline besprochen werden. Was wir damit erreichen wollen, ist, dass sich Zahnärzte mit ihrem Steuer- oder Wirtschaftsberater auf Augenhöhe unterhalten können. Das ist bisher sehr gut angelaufen.

Mit FVDZ-Control-doc bieten wir über unseren Kooperationspartner, der FIBU-doc Praxismanagement GmbH, ein Instrument, mit dem jeder Zahnarzt seine eigenen betriebswirtschaftlichen wichtigen Kennzahlen ganz leicht im Blick behalten und sich, wenn er dies will, über ein Benchmark-System vergleichen kann. Das ist ein exklusives Angebot, welches es nur für FVDZMitglieder gibt.

Auch das Praxishandbuch wird derzeit auf die modernen Anforderungen hin überarbeitet und wird zukünftig in einer zeitgemäßen digitalen Ausgabe vorliegen.

Ein weiterer Schwerpunkt im Bereich Ökonomie ist das Thema Praxisabgabe und Praxisübernahme, denn in dem Fall geht es neben allen rechtlichen Fragen auch um viel Geld - sowohl in die eine als auch in andere Richtung. Auch hier will der FVDZ das nötige Know-how vermitteln und die entsprechenden Partner zusammenbringen.

\section{Was ist das Ziel für die nächste Wahlperiode?}

Unser Ziel als Verband ist es, die Inhaber einer freiberuflichen Praxis ganz praktisch zu unterstützen - und zwar unabhängig von verbandseigenen ökonomischen Interessen, was ja bei Banken, Versicherungen und Beraterfirmen, die sich im Bereich Praxismanagement mit vielfältigen Angeboten tummeln, nicht immer der Fall ist. Unser Augenmerk wird da sicherlich weiterhin und verstärkt auf dem Thema Praxisabgabe und Praxisübernahme liegen. Es ist festzustellen, dass jüngere Zahnärzte eine längere Findungsphase haben, bis sie sich für eine eigene Niederlassung entscheiden. In diesem Prozess wollen wir weiter fachkundig begleiten. Das Thema Ökonomie ist eines, das man sich selbst erarbeiten muss, weil man an der Hochschule dafür nicht ausgebildet wird. Das heißt, dass wir die Angebote für Praxisinhaber und vor allem Berufseinsteiger weiter ausbauen wollen, denn das Potenzial, das wir durch unseren studentischen Zuwachs im FVDZ gewonnen haben, gilt es mitzunehmen in die nächste berufliche Phase. Da werden an den Verband hohe Anforderungen gestellt. Die Grundlagen sind gelegt, daran werden wir weiterarbeiten.

Unsere Mitglieder werden mit dieser berufspolitisch wichtigen Arbeit auf dem Gebiet der Ökonomie in der Ausübung ihrer Freiberuflichkeit gestärkt.

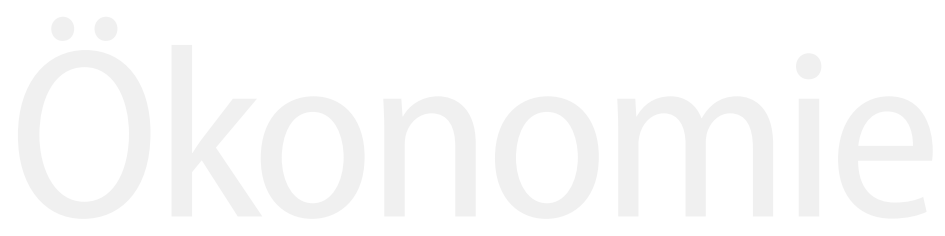

